\title{
GAIA Level 1 Ectopic Pregnancy
}

National Cancer Institute

\section{Source}

National Cancer Institute. GAIA Level 1 Ectopic Pregnancy. NCI Thesaurus. Code C128710.

GAIA Level 1 Ectopic Pregnancy is defined by either of the following two criteria: first, the following three requirements must be met: a) Gestational age is within the pre-defined range for the selected ectopic pregnancy definition as assessed by maternal and/or fetal parameters (Level 1-2) (Brighton Preterm Birth Gestational Age algorithm; b) B-HCG serum blood test greater than $2000 \mathrm{mlU} / \mathrm{ml}$; c) Transvaginal ultrasound showing no intrauterine pregnancy; OR second, the following two criteria must be met: a) Gestational age is within the pre-defined range for the selected ectopic pregnancy definition as assessed by maternal and/or fetal parameters (Level 1-2) (Brighton Preterm Birth Gestational Age algorithm; b) Transvaginal ultrasound showing extrauterine pregnancy OR no products of conception found on endometrial curettage after dilation and curettage procedure. 